\title{
Boundary perturbations due to the presence of small linear cracks in an elastic body*
}

\author{
Habib Ammari ${ }^{\dagger} \quad$ Hyeonbae Kang ${ }^{\ddagger} \quad$ Hyundae Lee Jisun Lim $^{\ddagger}$
}

April 9, 2018

\begin{abstract}
In this paper, Neumann cracks in elastic bodies are considered. We establish a rigorous asymptotic expansion for the boundary perturbations of the displacement (and traction) vectors that are due to the presence of a small elastic linear crack. The formula reveals that the leading order term is $\varepsilon^{2}$ where $\varepsilon$ is the length of the crack, and the $\varepsilon^{3}$-term vanishes. We obtain an asymptotic expansion of the elastic potential energy as an immediate consequence of the boundary perturbation formula. The derivation is based on layer potential techniques. It is expected that the formula would lead to very effective direct approaches for locating a collection of small elastic cracks and estimating their sizes and orientations.
\end{abstract}

Mathematics subject classification (MSC2000): 35B30, 74B05

Keywords: elastic crack, expansion formula, boundary perturbations

\section{Introduction}

The displacement (or traction) vector can be perturbed due to the presence of a small crack in an elastic medium. The aim of this paper is to derive an asymptotic formula for the boundary perturbations of the displacement as the length of the crack tends to zero. The focus is on cracks with homogeneous Neumann boundary conditions, i.e., perfectly insulating cracks. We consider the linear isotropic elasticity system in two dimensions and assume that the crack is a line segment of small size. The derivation of the asymptotic formula is based on layer potential techniques.

The paper extends recent asymptotic results that have been used for an efficient imaging of small defects. In [8] an electrostatic model, where the crack is perfectly conducting, was considered and an asymptotic expansion of the boundary perturbations that are due to the presence of a small linear crack was derived. The asymptotic formula leads us to efficient algorithms to detect cracks using boundary measurements [3, 8]. Their resolution and stability of the algorithms with respect to medium and measurement noises

${ }^{*}$ This work was supported by the ERC Advanced Grant Project MULTIMOD-267184 and NRF grants No. 2009-0090250, 2010-0004091, and 2010-0017532.

${ }^{\dagger}$ Department of Mathematics and Applications, Ecole Normale Supérieure, 45 Rue d’Ulm, 75005 Paris, France (habib.ammari@ens.fr).

${ }^{\ddagger}$ Department of Mathematics, Inha University, Incheon 402-751, Korea (hbkang@inha.ac.kr, hdlee@inha.ac.kr, jisunlim@inha.ac.kr). 
were investigated in 2, 4. There were some work on boundary perturbation due to the presence of small inclusions in linear elasticity; the effect of small inclusions on boundary measurements has been studied in [5, 9]. The effect of thin elastic inclusions on boundary measurements was quantified in [10, 12]. Direct reconstruction algorithms for locating small or thin elastic defects were developed in [1, 11, 15, 16]. We emphasize that the results of this paper (on cracks) can not be obtained as a limiting case of thin inclusions.

The results of this paper reveals that the leading order term of the boundary perturbation is $\varepsilon^{2}$ where $\varepsilon$ is the length of the crack, and its intensity is given by the traction force of the background solution on the crack (see Theorem 4.1.) We also prove that the $\varepsilon^{3}$-order term vanishes. By integrating the boundary perturbation formula against the given traction, we are able to derive an asymptotic expansion for the perturbation of the elastic potential energy, which is an improvement over already existing results [26, 22] (see the discussion at the end of Section 5).

The boundary perturbation formula derived in this paper carries information about the location, size, and orientation of the crack, and we expect, as in the electrostatic case, that the formula will provide a powerful tool to solve the inverse problem of identifying the cracks in terms of boundary measurements. The implementation of imaging algorithms based on the present expansion and the analysis of their resolution and stability will be the subject of a forthcoming paper.

The paper is organized as follows. In section 2, a representation formula for the solution of the problem in the presence of a Neumann crack is derived. Section 3 is devoted to making explicit the hyper-singular character involved in the representation formula. Using analytical results for the finite Hilbert transform, we derive in section 4 an asymptotic expansion of the effect of a small Neumann crack on the boundary values of the solution. Section [5 aims to derive the topological derivative of the elastic potential energy functional. Appendix contains technical calculation of the double layer potential.

\section{A representation formula}

Let $\Omega \subset \mathbb{R}^{2}$ be an open bounded domain, whose boundary $\partial \Omega$ is of class $\mathcal{C}^{1, \alpha}$ for some $\alpha>0$. We assume that $\Omega$ is a homogeneous isotropic elastic body so that its elasticity tensor $\mathbb{C}=\left(C_{i j k l}\right)$ is given by

$$
C_{i j k l}=\lambda \delta_{i j} \delta_{k l}+\mu\left(\delta_{i k} \delta_{j l}+\delta_{i l} \delta_{j k}\right)
$$

with the Lamé coefficients $\lambda$ and $\mu$ satisfying $\mu>0$ and $\lambda+\mu>0$. Let $\gamma_{\varepsilon} \subset \Omega$ be a small straight crack with size $\varepsilon$, located at some fixed distance $d_{0}$ from $\partial \Omega$, i.e.,

$$
\operatorname{dist}\left(\gamma_{\varepsilon}, \partial \Omega\right) \geq d_{0} .
$$

We denote by $\mathbf{e}^{\perp}$ a unit normal to $\gamma_{\varepsilon}$.

Let

$$
\Psi:=\left\{\psi: \partial_{i} \psi_{j}+\partial_{j} \psi_{i}=0, \quad 1 \leq i, j \leq 2\right\},
$$

or equivalently,

$$
\Psi=\operatorname{span}\left\{\left[\begin{array}{l}
1 \\
0
\end{array}\right],\left[\begin{array}{l}
0 \\
1
\end{array}\right],\left[\begin{array}{c}
y \\
-x
\end{array}\right]\right\}
$$


Introduce the space

$$
L_{\Psi}^{2}(\partial \Omega):=\left\{\mathbf{f} \in L^{2}(\partial \Omega): \int_{\partial \Omega} \mathbf{f} \cdot \psi d \sigma=0 \quad \text { for all } \psi \in \Psi\right\} .
$$

Let $\mathbf{u}_{\varepsilon}$ be the displacement vector caused by the traction $\mathbf{g} \in L_{\Psi}^{2}(\partial \Omega)$ applied on the boundary $\partial \Omega$ in the presence of $\gamma_{\varepsilon}$. Then $\mathbf{u}_{\varepsilon}$ is the solution to

$$
\begin{cases}\nabla \cdot \sigma\left(\mathbf{u}_{\varepsilon}\right)=0 & \text { in } \Omega \backslash \bar{\gamma}_{\varepsilon} \\ \sigma\left(\mathbf{u}_{\varepsilon}\right) \mathbf{n}=\mathbf{g} & \text { on } \partial \Omega, \\ \sigma\left(\mathbf{u}_{\varepsilon}\right) \mathbf{e}^{\perp}=0 & \text { on } \gamma_{\varepsilon}\end{cases}
$$

where $\mathbf{n}$ is the outward unit normal to $\partial \Omega$ and $\sigma\left(\mathbf{u}_{\varepsilon}\right)$ is the stress defined by

$$
\sigma\left(\mathbf{u}_{\varepsilon}\right)=\mathbb{C} \nabla^{s} \mathbf{u}_{\varepsilon}:=\frac{1}{2} \mathbb{C}\left(\nabla \mathbf{u}_{\varepsilon}+\nabla \mathbf{u}_{\varepsilon}^{T}\right) .
$$

Here, $\nabla^{s} \mathbf{u}_{\varepsilon}=\frac{1}{2}\left(\nabla \mathbf{u}_{\varepsilon}+\nabla \mathbf{u}_{\varepsilon}^{T}\right)$ is the strain tensor and the superscript $T$ denotes the transpose of a matrix. Note that the functions in $\Psi$ are solutions to the homogeneous problem (2.2) with $\mathbf{g}=0$. So we impose orthogonality condition on $\mathbf{u}_{\varepsilon}$ to guarantee the uniqueness of a solution to (2.2):

$$
\int_{\partial \Omega} \mathbf{u}_{\varepsilon} \cdot \psi d \sigma=0 \quad \text { for all } \psi \in \Psi .
$$

Let $\mathbf{u}_{0}$ be the solution in the absence of the crack, i.e., the solution to

$$
\begin{cases}\nabla \cdot \sigma\left(\mathbf{u}_{0}\right)=0 & \text { in } \Omega, \\ \sigma\left(\mathbf{u}_{0}\right) \mathbf{n}=\mathbf{g} & \text { on } \partial \Omega,\end{cases}
$$

with the orthogonality condition: $\left.\mathbf{u}_{0}\right|_{\partial \Omega} \in L_{\Psi}^{2}\left(\partial \Omega\right.$ ) (or equivalently, (2.4) with $\mathbf{u}_{\varepsilon}$ replaced with $\mathbf{u}_{0}$ ).

It is well-known that the solution $\mathbf{u}_{\varepsilon}$ to (2.2) belongs to $H^{1}\left(\Omega \backslash \gamma_{\varepsilon}\right)$. In fact, we have

$$
\left\|\mathbf{u}_{\varepsilon}\right\|_{H^{1}\left(\Omega \backslash \gamma_{\varepsilon}\right)} \leq C
$$

for some $C$ independent of $\varepsilon$. To see this, we introduce the potential energy functional

$$
J_{\varepsilon}[\mathbf{u}]:=-\frac{1}{2} \int_{\Omega \backslash \gamma_{\varepsilon}} \sigma(\mathbf{u}): \nabla^{s} \mathbf{u} .
$$

The solution $\mathbf{u}_{\varepsilon}$ of $(2.2)$ is the maximizer of $J_{\varepsilon}$, i.e.,

$$
J_{\varepsilon}\left[\mathbf{u}_{\varepsilon}\right]=\max J_{\varepsilon}[\mathbf{u}]
$$

where the maximum is taken over all $\mathbf{u} \in H^{1}\left(\Omega \backslash \gamma_{\varepsilon}\right)$ satisfying $\sigma(\mathbf{u}) \mathbf{n}=\mathbf{g}$ on $\partial \Omega$ and $\sigma(\mathbf{u}) \mathbf{e}^{\perp}=0$ on $\gamma_{\varepsilon}$. Let $\mathbf{v}$ be a smooth function with a compact support in $\Omega$ such that $\sigma(\mathbf{v}) \mathbf{e}^{\perp}=-\sigma\left(\mathbf{u}_{0}\right) \mathbf{e}^{\perp}$ on $\gamma_{\varepsilon}$. We may choose $\mathbf{v}$ so that $J_{\varepsilon}[\mathbf{v}]$ is independent of $\varepsilon$. Since $0 \geq J_{\varepsilon}\left[\mathbf{u}_{\varepsilon}\right] \geq J_{\varepsilon}\left[\mathbf{u}_{0}+\mathbf{v}\right]$, we have

$$
\left\|\nabla^{s} \mathbf{u}_{\varepsilon}\right\|_{L^{2}\left(\Omega \backslash \gamma_{\varepsilon}\right)} \leq C .
$$


We then have from the Korn's inequality that there is a constant $C$ independent of $\varepsilon$ such that

$$
\left\|\mathbf{u}_{\varepsilon}-\mathbf{u}_{0}\right\|_{H^{1}\left(\Omega \backslash \gamma_{\varepsilon}\right)} \leq C\left(\left\|\nabla^{s}\left(\mathbf{u}_{\varepsilon}-\mathbf{u}_{0}\right)\right\|_{L^{2}\left(\Omega \backslash \gamma_{\varepsilon}\right)}+\left\|\mathbf{u}_{\varepsilon}-\mathbf{u}_{0}\right\|_{H^{1 / 2}(\partial \Omega)}\right) .
$$

Since $\left\|\mathbf{u}_{\varepsilon}-\mathbf{u}_{0}\right\|_{H^{1 / 2}(\partial \Omega)}$ is bounded regardless of $\varepsilon$ as we shall show later (Theorem 4.1), we obtain (2.6).

Let

$$
\varphi_{\varepsilon}(\mathbf{x}):=\left.\mathbf{u}_{\varepsilon}\right|_{+}(\mathbf{x})-\left.\mathbf{u}_{\varepsilon}\right|_{-}(\mathbf{x}), \quad \mathbf{x} \in \gamma_{\varepsilon}
$$

where + (resp. - ) indicates the limit on the crack $\gamma_{\varepsilon}$ from the given normal direction $\mathbf{e}^{\perp}$ (resp. opposite direction), i.e.,

$$
\mathbf{u}_{ \pm}(x):=\lim _{t \rightarrow 0} \mathbf{u}\left(\mathbf{x} \pm t \mathbf{e}^{\perp}\right) .
$$

We sometimes denote $\sigma(\mathbf{u}) \mathbf{n}$, the traction on $\partial \Omega$ (or on $\gamma_{\varepsilon}$ ), by $\partial \mathbf{u} / \partial \nu$, i.e.,

$$
\frac{\partial \mathbf{u}}{\partial \nu}:=\lambda(\nabla \cdot \mathbf{u}) \mathbf{n}+\mu\left(\nabla \mathbf{u}+\nabla \mathbf{u}^{T}\right) \mathbf{n} \quad \text { on } \partial \Omega .
$$

If $\Phi=\left(\Phi_{i j}\right)_{2 \times 2}$ is the Kelvin matrix of the fundamental solutions of Lamé system, i.e.,

$$
\Phi_{i j}(\mathbf{x}):=\frac{A}{2 \pi} \delta_{i j} \log |\mathbf{x}|-\frac{B}{2 \pi} \frac{x_{i} x_{j}}{|\mathbf{x}|^{2}}, \quad \mathbf{x} \neq 0 \in \mathbb{R}^{2},
$$

where

$$
A=\frac{\lambda+3 \mu}{2 \mu(\lambda+2 \mu)} \quad \text { and } \quad B=\frac{\lambda+\mu}{2 \mu(\lambda+2 \mu)},
$$

then the solution $\mathbf{u}_{\varepsilon}$ to (2.2) is represented as

$$
\begin{aligned}
\mathbf{u}_{\varepsilon}(\mathbf{x})= & \int_{\partial \Omega} \frac{\partial \Phi}{\partial \nu_{\mathbf{y}}}(\mathbf{x}-\mathbf{y}) \mathbf{u}_{\varepsilon}(\mathbf{y}) d \sigma(\mathbf{y})-\int_{\partial \Omega} \Phi(\mathbf{x}-\mathbf{y}) \frac{\partial \mathbf{u}_{\varepsilon}}{\partial \nu}(\mathbf{y}) d \sigma(\mathbf{y}) \\
& -\int_{\gamma_{\varepsilon}} \frac{\partial \Phi}{\partial \nu_{\mathbf{y}}}(\mathbf{x}-\mathbf{y}) \varphi_{\varepsilon}(\mathbf{y}) d \sigma(\mathbf{y}), \quad \mathbf{x} \in \Omega \backslash \gamma_{\varepsilon} .
\end{aligned}
$$

The solution $\mathbf{u}_{0}$ to (2.5) is represented as

$$
\mathbf{u}_{0}(\mathbf{x})=\int_{\partial \Omega} \frac{\partial \Phi}{\partial \nu_{\mathbf{y}}}(\mathbf{x}-\mathbf{y}) \mathbf{u}_{0}(\mathbf{y}) d \sigma(\mathbf{y})-\int_{\partial \Omega} \Phi(\mathbf{x}-\mathbf{y}) \frac{\partial \mathbf{u}_{0}}{\partial \nu}(\mathbf{y}) d \sigma(\mathbf{y}) .
$$

Let

$$
\mathbf{w}_{\varepsilon}:=\mathbf{u}_{\varepsilon}-\mathbf{u}_{0} .
$$

Since $\frac{\partial \mathbf{u}_{\varepsilon}}{\partial \nu}=\frac{\partial \mathbf{u}_{0}}{\partial \nu}$ on $\partial \Omega$, by subtracting above two identities, we have

$$
\mathbf{w}_{\varepsilon}(\mathbf{x})-\mathcal{D}_{\Omega}\left[\mathbf{w}_{\varepsilon}\right](\mathbf{x})=-\mathcal{D}_{\varepsilon}\left[\varphi_{\varepsilon}\right](\mathbf{x}), \quad \mathbf{x} \in \Omega,
$$

where the double layer potentials $\mathcal{D}_{\Omega}$ and $\mathcal{D}_{\varepsilon}$ are defined by

$$
\mathcal{D}_{\Omega}\left[\mathbf{w}_{\varepsilon}\right](\mathbf{x}):=\int_{\partial \Omega} \frac{\partial \Phi}{\partial \nu_{\mathbf{y}}}(\mathbf{x}-\mathbf{y}) \mathbf{w}_{\varepsilon}(\mathbf{y}) d \sigma(\mathbf{y}), \quad \mathbf{x} \in \Omega
$$


and

$$
\mathcal{D}_{\varepsilon}\left[\varphi_{\varepsilon}\right](\mathbf{x}):=\int_{\gamma_{\varepsilon}} \frac{\partial \Phi}{\partial \nu_{\mathbf{y}}}(\mathbf{x}-\mathbf{y}) \varphi_{\varepsilon}(\mathbf{y}) d \sigma(\mathbf{y}), \quad \mathbf{x} \in \Omega .
$$

Let $\mathbf{n}_{\mathbf{x}}$ denote the outward normal to $\partial \Omega$ at $\mathbf{x} \in \partial \Omega$ and let

$$
\left.\mathcal{D}_{\Omega}[\varphi]\right|_{-}(\mathbf{x})=\lim _{t \rightarrow 0+} \mathcal{D}_{\Omega}[\varphi]\left(\mathbf{x}+t \mathbf{n}_{\mathbf{x}}\right) .
$$

Then, it is well known (see, for example, [6]) that

$$
\left.\mathcal{D}_{\Omega}[\varphi]\right|_{-}(\mathbf{x})=\left(\frac{1}{2} I+\mathcal{K}_{\Omega}\right)[\varphi](\mathbf{x}), \quad \text { a.e. } \mathbf{x} \in \partial \Omega,
$$

where $\mathcal{K}_{\Omega}$ is the boundary integral operator defined by

$$
\mathcal{K}_{\Omega}[\varphi](\mathbf{x}):=\text { p.v. } \int_{\partial \Omega} \frac{\partial \Phi}{\partial \nu_{\mathbf{y}}}(\mathbf{x}-\mathbf{y}) \mathbf{w}_{\varepsilon}(\mathbf{y}) d \sigma(\mathbf{y}), \quad \mathbf{x} \in \partial \Omega,
$$

and $I$ is the identity operator. Here, p.v. stands for the Cauchy principal value. It then follows from (2.16) that

$$
\left(-\frac{1}{2} I+\mathcal{K}_{\Omega}\right)\left[\mathbf{w}_{\varepsilon}\right](\mathbf{x})=\mathcal{D}_{\varepsilon}\left[\varphi_{\varepsilon}\right](\mathbf{x}), \quad \mathbf{x} \in \partial \Omega .
$$

Since $-\frac{1}{2} I+\mathcal{K}_{\Omega}$ is invertible on $L_{\Psi}^{2}(\partial \Omega)$ (see, for instance, [13]), we have

$$
\mathbf{w}_{\varepsilon}(\mathbf{x})=\int_{\gamma_{\varepsilon}} \frac{\partial}{\partial \nu_{\mathbf{y}}}\left(-\frac{1}{2} I+\mathcal{K}_{\Omega}\right)^{-1}[\Phi(\cdot-\mathbf{y})](\mathbf{x}) \varphi_{\varepsilon}(\mathbf{y}) d \sigma(\mathbf{y}), \quad \mathbf{x} \in \partial \Omega .
$$

Note that

$$
\left(-\frac{1}{2} I+\mathcal{K}_{\Omega}\right)^{-1}[\Phi(\cdot-\mathbf{y})](\mathbf{x})=\mathbf{N}(\mathbf{x}, \mathbf{y}), \quad \mathbf{x} \in \partial \Omega, \quad \mathbf{y} \in \Omega
$$

modulo a function in $\Psi$, where $\mathbf{N}(\mathbf{x}, \mathbf{y})$ is the Neumann function for the Lamé system on $\Omega$, namely, for $\mathbf{y} \in \Omega, \mathbf{N}(\mathbf{x}, \mathbf{y})$ is the solution to

$$
\begin{cases}\nabla \cdot \sigma(\mathbf{N}(\cdot, \mathbf{y}))=-\delta_{\mathbf{y}} \mathbf{I} & \text { in } \Omega, \\ \sigma(\mathbf{N}(\cdot, \mathbf{y})) \mathbf{n}=-\frac{1}{|\partial \Omega|} \mathbf{I} & \text { on } \partial \Omega,\end{cases}
$$

subject to the orthogonality condition:

$$
\int_{\partial \Omega} \mathbf{N}(\mathbf{x}, \mathbf{y}) \cdot \psi(\mathbf{x}) d \sigma(\mathbf{x})=0 \quad \text { for all } \psi \in \Psi .
$$

Here, $\mathbf{I}$ is the 2 identity matrix. See [6, 7, 17] for properties of the Neumann function and a proof of (2.21). Thus we obtain from (2.20) that

$$
\mathbf{u}_{\varepsilon}(\mathbf{x})=\mathbf{u}_{0}(\mathbf{x})+\int_{\gamma_{\varepsilon}} \frac{\partial}{\partial \nu_{\mathbf{y}}} \mathbf{N}(\mathbf{x}, \mathbf{y}) \varphi_{\varepsilon}(\mathbf{y}) d \sigma(\mathbf{y}), \quad \mathbf{x} \in \partial \Omega .
$$

We now describe the scheme to derive an asymptotic expansion of $\mathbf{u}_{\epsilon}-\mathbf{u}_{0}$ on $\partial \Omega$. Since $\frac{\partial \mathbf{u}_{\epsilon}}{\partial \nu}=\sigma\left(\mathbf{u}_{\epsilon}\right) \mathbf{e}^{\perp}=0$ on $\gamma_{\varepsilon}$, we use (2.16) to obtain

$$
\frac{\partial \mathbf{u}_{0}}{\partial \nu}+\frac{\partial}{\partial \nu} \mathcal{D}_{\Omega}\left[\mathbf{w}_{\varepsilon}\right]=\frac{\partial}{\partial \nu} \mathcal{D}_{\varepsilon}\left[\varphi_{\varepsilon}\right] \quad \text { on } \gamma_{\epsilon} .
$$

We solve this integral equation for $\varphi_{\varepsilon}$ and then substitute it into (2.23) to derive an asymptotic expansion of $\mathbf{u}_{\varepsilon}$ as $\varepsilon \rightarrow 0$. 


\section{Derivation of an explicit integral equation}

In view of (2.24), we need to compute $\frac{\partial}{\partial \nu_{\mathbf{x}}}\left(\frac{\partial \Phi}{\partial \nu_{\mathbf{y}}}(\mathbf{x}-\mathbf{y})\right)$ on $\gamma_{\varepsilon}$. As before $\mathbf{e}^{\perp}$ is the unit normal to $\gamma_{\varepsilon}$, and denoted by $\mathbf{e}^{\perp}=\left(n_{1}, n_{2}\right)$. It is worth mentioning that $n_{1}$ and $n_{2}$ are constant since $\gamma_{\varepsilon}$ is a line segment. It is convenient to use the following expression of the conormal derivative:

$$
\frac{\partial \mathbf{u}}{\partial \nu}=T(\partial) \mathbf{u}
$$

where the operator $T(\partial)=T\left(\partial_{1}, \partial_{2}\right)$, where $\partial_{j}=\frac{\partial}{\partial x_{j}}$, is defined by

$$
T\left(\xi_{1}, \xi_{2}\right):=\left[\begin{array}{cc}
(\lambda+2 \mu) n_{1} \xi_{1}+\mu n_{2} \xi_{2} & \mu n_{2} \xi_{1}+\lambda n_{1} \xi_{2} \\
\lambda n_{2} \xi_{1}+\mu n_{1} \xi_{2} & \mu n_{1} \xi_{1}+(\lambda+2 \mu) n_{2} \xi_{2}
\end{array}\right] .
$$

We first obtain the following formula whose derivation will be given in Appendix A. For $\mathbf{x} \neq \mathbf{y}$, we have

$$
\begin{gathered}
\left(\frac{\partial \Phi}{\partial \nu_{\mathbf{y}}}(\mathbf{x}-\mathbf{y})\right)_{i j}=\left[a \delta_{i j}+b \frac{\left(x_{i}-y_{i}\right)\left(x_{j}-y_{j}\right)}{|\mathbf{x}-\mathbf{y}|^{2}}\right] \sum_{l=1}^{2} \frac{n_{l}\left(x_{l}-y_{l}\right)}{|\mathbf{x}-\mathbf{y}|^{2}} \\
-a \frac{n_{j}\left(x_{i}-y_{i}\right)-n_{i}\left(x_{j}-y_{j}\right)}{|\mathbf{x}-\mathbf{y}|^{2}}
\end{gathered}
$$

where

$$
a=-\frac{\mu}{2 \pi(\lambda+2 \mu)}, \quad b=-\frac{(\lambda+\mu)}{\pi(\lambda+2 \mu)} .
$$

Let $v_{i j}=\left(\frac{\partial \Phi}{\partial \nu_{\mathbf{y}}}(\mathbf{x}-\mathbf{y})\right)_{i j}$ for convenience and let

$$
W(\mathbf{x}-\mathbf{y}):=\frac{\partial}{\partial \nu_{\mathbf{x}}}\left(\frac{\partial \Phi}{\partial \nu_{\mathbf{y}}}(\mathbf{x}-\mathbf{y})\right)
$$

Then one can use (3.2) to derive

$$
\begin{aligned}
& W(\mathbf{x}-\mathbf{y})_{11}=n_{1}\left[(\lambda+2 \mu) \partial_{1} v_{11}+\lambda \partial_{2} v_{21}\right]+n_{2}\left(\mu \partial_{2} v_{11}+\mu \partial_{1} v_{21}\right), \\
& W(\mathbf{x}-\mathbf{y})_{12}=n_{1}\left[(\lambda+2 \mu) \partial_{1} v_{12}+\lambda \partial_{x_{2}} v_{22}\right]+n_{2}\left(\mu \partial_{2} v_{12}+\mu \partial_{1} v_{22}\right), \\
& W(\mathbf{x}-\mathbf{y})_{21}=n_{1}\left(\mu \partial_{2} v_{11}+\mu \partial_{1} v_{21}\right)+n_{2}\left[\lambda \partial_{1} v_{11}+(\lambda+2 \mu) \partial_{2} v_{21}\right] \\
& W(\mathbf{x}-\mathbf{y})_{22}=n_{1}\left(\mu \partial_{2} v_{12}+\mu \partial_{1} v_{22}\right)+n_{2}\left[\lambda \partial_{1} v_{12}+(\lambda+2 \mu) \partial_{2} v_{22}\right]
\end{aligned}
$$

Since the crack which we consider is a line segment with length $\varepsilon$ in the domain $\Omega \subset \mathbb{R}^{2}$, we may assume, after rotation and translation if necessary, that it is given by

$$
\gamma_{\varepsilon}=\left\{\left(x_{1}, 0\right):-\varepsilon / 2 \leq x_{1} \leq \varepsilon / 2\right\}
$$


In this case, one can check that

$$
\begin{aligned}
& \partial_{2} v_{11}=\left(a+b \frac{\left(x_{1}-y_{1}\right)^{2}}{|\mathbf{x}-\mathbf{y}|^{2}}\right) \frac{1}{|\mathbf{x}-\mathbf{y}|^{2}}=\frac{a+b}{\left(x_{1}-y_{1}\right)^{2}} \\
& \partial_{1} v_{21}=a\left(\frac{1}{|\mathbf{x}-\mathbf{y}|^{2}}-2 \frac{x_{1}-y_{1}}{|\mathbf{x}-\mathbf{y}|^{2}} \frac{x_{1}-y_{1}}{\left|x_{1}-y_{1}\right|^{2}}\right)=-\frac{a}{\left(x_{1}-y_{1}\right)^{2}}, \\
& \partial_{1} v_{12}=-a\left(\frac{1}{|\mathbf{x}-\mathbf{y}|^{2}}-2 \frac{x_{1}-y_{1}}{|\mathbf{x}-\mathbf{y}|^{2}} \frac{x_{1}-y_{1}}{\left|x_{1}-y_{1}\right|^{2}}\right)=\frac{a}{\left(x_{1}-y_{1}\right)^{2}}, \\
& \partial_{2} v_{22}=\frac{a}{\left(x_{1}-y_{1}\right)^{2}} \\
& \partial_{1} v_{11}=\partial_{2} v_{21}=\partial_{2} v_{12}=\partial_{1} v_{22}=0 .
\end{aligned}
$$

Since $\mathbf{e}^{\perp}=\left(n_{1}, n_{2}\right)=(0,1)$, we have

$$
\begin{aligned}
W(\mathbf{x}-\mathbf{y})_{11} & =\mu \partial_{2} v_{11}+\mu \partial_{1} v_{21} \\
& =\mu(a+b) \frac{1}{\left(x_{1}-y_{1}\right)^{2}}-\mu a \frac{1}{\left(x_{1}-y_{1}\right)^{2}}=\frac{\mu b}{\left(x_{1}-y_{1}\right)^{2}}, \\
W(\mathbf{x}-\mathbf{y})_{12} & =\mu \partial_{2} v_{12}+\mu \partial_{1} v_{22}=0 \\
W(\mathbf{x}-\mathbf{y})_{21} & =\lambda \partial_{1} v_{11}+(\lambda+2 \mu) \partial_{2} v_{21}=0 \\
W(\mathbf{x}-\mathbf{y})_{22} & =\lambda \partial_{1} v_{12}+(\lambda+2 \mu) \partial_{2} v_{22} \\
& =\lambda a \frac{1}{\left(x_{1}-y_{1}\right)^{2}}+(\lambda+2 \mu) a \frac{1}{\left(x_{1}-y_{1}\right)^{2}}=\frac{2(\lambda+\mu) a}{\left(x_{1}-y_{1}\right)^{2}}
\end{aligned}
$$

that is,

$$
W(\mathbf{x}-\mathbf{y})=\frac{1}{\left(x_{1}-y_{1}\right)^{2}}\left[\begin{array}{cc}
-\frac{\mu(\lambda+\mu)}{\pi(\lambda+2 \mu)} & 0 \\
0 & -\frac{\mu(\lambda+\mu)}{\pi(\lambda+2 \mu)}
\end{array}\right] .
$$

Note that

$$
\frac{\mu(\lambda+\mu)}{\lambda+2 \mu}=\frac{E}{4}
$$

where $E$ is the Young's modulus in two dimensions. So, we have

$$
W(\mathbf{x}-\mathbf{y})=-\frac{E}{4 \pi} \frac{1}{\left(x_{1}-y_{1}\right)^{2}} \mathbf{I} .
$$

So far we have shown that if $\gamma_{\varepsilon}$ is given by (3.6), then

$$
\frac{\partial}{\partial \nu_{\mathbf{x}}} \mathcal{D}_{\varepsilon}\left[\varphi_{\varepsilon}\right](\mathbf{x})=-\frac{E}{4 \pi} \int_{-\varepsilon / 2}^{\varepsilon / 2} \frac{\varphi_{\varepsilon}(y)}{(x-y)^{2}} d y, \quad \mathbf{x}=(x, 0),-\varepsilon / 2<x<\varepsilon / 2 .
$$

Here the integral is hyper-singular and should be understood as a finite part in the sense of Hadamard, which will be defined in the next section. So the integral equation (2.24) becomes

$$
\frac{1}{\pi} \int_{-\varepsilon / 2}^{\varepsilon / 2} \frac{\varphi_{\varepsilon}(y)}{(x-y)^{2}} d y=-\frac{4}{E} \mathbf{f}(x), \quad-\varepsilon / 2<x<\varepsilon / 2,
$$

where

$$
\mathbf{f}(x)=\frac{\partial \mathbf{u}_{0}}{\partial \nu}(x, 0)+\frac{\partial}{\partial \nu} \mathcal{D}_{\Omega}\left[\mathbf{w}_{\varepsilon}\right](x, 0) .
$$


Define

$$
\mathbf{f}_{\varepsilon}(x):=\mathbf{f}\left(\frac{\varepsilon}{2} x\right)
$$

and

$$
\psi_{\varepsilon}(x):=\frac{2}{\varepsilon} \varphi_{\varepsilon}\left(\frac{\varepsilon}{2} x\right), \quad-1<x<1 .
$$

Then the scaled integral equation is

$$
\frac{1}{\pi} \int_{-1}^{1} \frac{\psi_{\varepsilon}(y)}{(x-y)^{2}} d y=-\frac{4}{E} \mathbf{f}_{\varepsilon}(x), \quad-1<x<1,
$$

which we solve in the next section.

\section{Asymptotic expansion}

The integral in (3.15) is understood as a finite-part in the sense of Hadamard [19, 20]: for $\psi \in \mathcal{C}^{1, \alpha}(-1,1)(0<\alpha \leq 1)$

$$
f_{-1}^{1} \frac{\psi(y)}{(x-y)^{2}} d y=\lim _{\delta \rightarrow 0}\left[\int_{-1}^{x-\delta} \frac{\psi(y)}{(x-y)^{2}} d y+\int_{x+\delta}^{1} \frac{\psi(y)}{(x-y)^{2}} d y-\frac{2 \psi(x)}{\delta}\right] .
$$

Define

$$
\mathcal{A}[\psi](x):=\frac{1}{\pi} \mathcal{火}_{-1}^{1} \frac{\psi(y)}{(x-y)^{2}} d y, \quad|x|<1 .
$$

It is known $([14,[18])$ that

$$
\mathcal{A}[\psi](x)=-\frac{d}{d x} \mathcal{H}[\psi](x),
$$

where $\mathcal{H}$ is the (finite) Hilbert transform, i.e.,

$$
\mathcal{H}[\psi](x)=\text { p.v. } \frac{1}{\pi} \int_{-1}^{1} \frac{\psi(y)}{x-y} d y .
$$

More properties of finite-part integrals and principal-value integrals can be found in 17 , 19, 20, 21, 25, 27].

If $\psi(-1)=\psi(1)=0$, we have from (4.3) that

$$
\mathcal{A}[\psi](x)=-\mathcal{H}\left[\psi^{\prime}\right](x)
$$

Thus we can invert the operator $\mathcal{A}$ using the properties of $\mathcal{H}$. The set $\mathcal{Y}$, given by

$$
\mathcal{Y}=\left\{\varphi: \int_{-1}^{1} \sqrt{1-x^{2}}|\varphi(x)|^{2} d x<+\infty\right\}
$$

is a Hilbert space with the norm

$$
\|\varphi\|_{\mathcal{Y}}=\left(\int_{-1}^{1} \sqrt{1-x^{2}}|\varphi(x)|^{2} d x\right)^{1 / 2} .
$$


It is well known (see, for example, [7, section 5.2]) that $\mathcal{H}$ maps $\mathcal{Y}$ onto itself and its null space is the one dimensional space generated by $1 / \sqrt{1-x^{2}}$. Therefore, if we define

$$
\mathcal{X}=\left\{\psi \in \mathcal{C}^{0}([-1,1]): \psi^{\prime} \in \mathcal{Y}, \psi(-1)=\psi(1)=0\right\}
$$

where $\psi^{\prime}$ is the distributional derivative of $\psi$, then $\mathcal{A}: \mathcal{X} \rightarrow \mathcal{Y}$ is invertible. We note that $\mathcal{X}$ is a Banach space with the norm

$$
\|\psi\|_{\mathcal{X}}=\|\psi\|_{L^{\infty}}+\left\|\psi^{\prime}\right\|_{\mathcal{Y}}
$$

Using the Hilbert inversion formula (see, for example, [7, section 5.2]), we can check

$$
\begin{aligned}
& \mathcal{A}^{-1}[1](x)=-\sqrt{1-x^{2}}, \\
& \mathcal{A}^{-1}[y](x)=-\frac{x}{2} \sqrt{1-x^{2}} .
\end{aligned}
$$

The equation (3.15) can be written as

$$
\mathcal{A}\left[\psi_{\varepsilon}\right](x)=-\frac{4}{E} \mathbf{f}_{\varepsilon}(x), \quad-1<x<1 .
$$

The Taylor expansion yields

$$
\frac{\partial \mathbf{u}_{0}}{\partial \nu}\left(\frac{\varepsilon}{2} x, 0\right)=\frac{\partial \mathbf{u}_{0}}{\partial \nu}(0)+\frac{\varepsilon x}{2} \frac{\partial^{2} \mathbf{u}_{0}}{\partial t \partial \nu}(0)+e_{1}(x),
$$

where $\partial / \partial t$ denotes the tangential derivative on $\gamma_{\varepsilon}$. The remainder term $e_{1}$ satisfies

$$
\left|e_{1}(x)\right| \leq C \varepsilon^{2}|x|^{2}
$$

and in particular,

$$
\left\|e_{1}\right\|_{\mathcal{Y}} \leq C \varepsilon^{2}
$$

On the other hand, since

$$
\frac{\partial}{\partial \nu} \mathcal{D}_{\Omega}\left[\mathbf{w}_{\varepsilon}\right]\left(\frac{\varepsilon}{2} x, 0\right)=\int_{\partial \Omega} \frac{\partial^{2} \mathbf{N}}{\partial \nu_{\mathbf{x}} \partial \nu_{\mathbf{y}}}\left(\left(\frac{\varepsilon}{2} x, 0\right), \mathbf{y}\right) \mathbf{w}_{\varepsilon}(\mathbf{y}) d \sigma(\mathbf{y}),
$$

and $\gamma_{\varepsilon}$ is away from $\partial \Omega$, one can see that

$$
\left\|\frac{\partial}{\partial \nu} \mathcal{D}_{\Omega}\left[\mathbf{w}_{\varepsilon}\right]\left(\frac{\varepsilon}{2} \cdot, 0\right)\right\|_{\mathcal{Y}} \leq C\left\|\mathbf{w}_{\varepsilon}\right\|_{L^{\infty}(\partial \Omega)} .
$$

Therefore, we have

$$
\mathbf{f}_{\varepsilon}(x)=\frac{\partial \mathbf{u}_{0}}{\partial \nu}(0)+\frac{\varepsilon x}{2} \frac{\partial^{2} \mathbf{u}_{0}}{\partial t \partial \nu}(0)+e(x),
$$

where $e$ satisfies

$$
\|e\|_{\mathcal{Y}} \leq C\left(\varepsilon^{2}+\left\|\mathbf{w}_{\varepsilon}\right\|_{L^{\infty}(\partial \Omega)}\right)
$$


We now obtain from (4.10) that

$$
\psi_{\varepsilon}(x)=-\frac{4}{E}\left[\frac{\partial \mathbf{u}_{0}}{\partial \nu}(0) \mathcal{A}^{-1}[1](x)+\frac{\varepsilon}{2} \frac{\partial^{2} \mathbf{u}_{0}}{\partial t \partial \nu}(0) \mathcal{A}^{-1}[y](x)+\mathcal{A}^{-1}[e](x)\right] .
$$

Note that $\mathcal{E}_{1}(x)=\mathcal{A}^{-1}[e](x)$ satisfies

$$
\left\|\mathcal{E}_{1}\right\|_{\mathcal{X}} \leq C\|e\|_{\mathcal{Y}} \leq C\left(\varepsilon^{2}+\left\|\mathbf{w}_{\varepsilon}\right\|_{L^{\infty}(\partial \Omega)}\right),
$$

and in particular,

$$
\left\|\mathcal{E}_{1}\right\|_{L^{\infty}(-1,1)} \leq C\left(\varepsilon^{2}+\left\|\mathbf{w}_{\varepsilon}\right\|_{L^{\infty}(\partial \Omega)}\right) .
$$

It then follows from (4.8) and (4.9) that

$$
\psi_{\varepsilon}(x)=\frac{4}{E}\left[\frac{\partial \mathbf{u}_{0}}{\partial \nu}(0) \sqrt{1-x^{2}}+\frac{\varepsilon}{4} \frac{\partial^{2} \mathbf{u}_{0}}{\partial t \partial \nu}(0) x \sqrt{1-x^{2}}+\mathcal{E}_{1}(x)\right] .
$$

Thus we have from (3.14) that

$$
\varphi_{\varepsilon}(x)=\frac{2}{E}\left[\frac{\partial \mathbf{u}_{0}}{\partial \nu}(0) \sqrt{\varepsilon^{2}-4 x^{2}}+\frac{1}{2} \frac{\partial^{2} \mathbf{u}_{0}}{\partial t \partial \nu}(0) x \sqrt{\varepsilon^{2}-4 x^{2}}+\mathcal{E}(x)\right], \quad(x, 0) \in \gamma_{\varepsilon},
$$

where $\mathcal{E}(x)=\frac{\varepsilon}{2} \mathcal{E}_{1}\left(\frac{2}{\varepsilon} x\right)$ satisfies

$$
\|\mathcal{E}\|_{L^{\infty}\left(\gamma_{\varepsilon}\right)} \leq C \varepsilon\left(\varepsilon^{2}+\left\|\mathbf{w}_{\varepsilon}\right\|_{L^{\infty}(\partial \Omega)}\right) .
$$

Substituting (4.19) into (2.23) we obtain

$$
\begin{aligned}
\mathbf{w}_{\varepsilon}(\mathbf{x})= & \frac{2}{E} \int_{\gamma_{\varepsilon}} \frac{\partial}{\partial \nu_{\mathbf{y}}} \mathbf{N}(\mathbf{x},(y, 0)) \sqrt{\varepsilon^{2}-4 y^{2}} d y \frac{\partial \mathbf{u}_{0}}{\partial \nu}(0) \\
& +\frac{1}{E} \int_{\gamma_{\varepsilon}} \frac{\partial}{\partial \nu_{\mathbf{y}}} \mathbf{N}(\mathbf{x},(y, 0)) y \sqrt{\varepsilon^{2}-4 y^{2}} d y \frac{\partial^{2} \mathbf{u}_{0}}{\partial t \partial \nu}(0) \\
& +\frac{2}{E} \int_{\gamma_{\varepsilon}} \frac{\partial}{\partial \nu_{\mathbf{y}}} \mathbf{N}(\mathbf{x},(y, 0)) \mathcal{E}(y) d y:=I+I I+I I I, \quad \mathbf{x} \in \partial \Omega .
\end{aligned}
$$

Since

$$
\frac{\partial}{\partial \nu_{\mathbf{y}}} \mathbf{N}(\mathbf{x},(y, 0))=\frac{\partial}{\partial \nu_{\mathbf{y}}} \mathbf{N}(\mathbf{x}, 0)+\frac{\partial^{2}}{\partial t_{\mathbf{y}} \partial \nu_{\mathbf{y}}} \mathbf{N}(\mathbf{x}, 0) y+O\left(y^{2}\right)
$$

we have

$$
\begin{aligned}
\int_{\gamma_{\varepsilon}} \frac{\partial}{\partial \nu_{\mathbf{y}}} \mathbf{N}(\mathbf{x},(y, 0)) \sqrt{\varepsilon^{2}-4 y^{2}} d y= & \frac{\partial}{\partial \nu_{\mathbf{y}}} \mathbf{N}(\mathbf{x}, 0) \int_{\gamma_{\varepsilon}} \sqrt{\varepsilon^{2}-4 y^{2}} d y \\
& +\frac{\partial^{2}}{\partial t_{\mathbf{y}} \partial \nu_{\mathbf{y}}} \mathbf{N}(\mathbf{x}, 0) \int_{\gamma_{\varepsilon}} y \sqrt{\varepsilon^{2}-4 y^{2}} d y+O\left(\varepsilon^{4}\right) \\
= & \frac{\pi \varepsilon^{2}}{2} \frac{\partial}{\partial \nu_{\mathbf{y}}} \mathbf{N}(\mathbf{x}, 0)+O\left(\varepsilon^{4}\right)
\end{aligned}
$$


and hence

$$
I=\frac{\pi \varepsilon^{2}}{E} \frac{\partial}{\partial \nu_{\mathbf{y}}} \mathbf{N}(\mathbf{x}, 0) \frac{\partial \mathbf{u}_{0}}{\partial \nu}(0)+O\left(\varepsilon^{4}\right) .
$$

Here and throughout this paper, $O\left(\varepsilon^{4}\right)$ is in the sense of the uniform norm on $\partial \Omega$. Similarly, one can show that

$$
I I=O\left(\varepsilon^{4}\right) .
$$

So we obtain that

$$
\mathbf{w}_{\varepsilon}(\mathbf{x})=\frac{\pi \varepsilon^{2}}{E} \frac{\partial}{\partial \nu_{\mathbf{y}}} \mathbf{N}(\mathbf{x}, 0) \frac{\partial \mathbf{u}_{0}}{\partial \nu}(0)+O\left(\varepsilon^{4}\right)+I I I .
$$

In particular, we have

$$
\left\|\mathbf{w}_{\varepsilon}\right\|_{L^{\infty}(\partial \Omega)} \leq C\left(\varepsilon^{2}+|I I I|\right) .
$$

But, because of (4.20), we arrive at

$$
|I I I| \leq C \varepsilon\|\mathcal{E}\|_{L^{\infty}\left(\gamma_{\varepsilon}\right)} \leq C \varepsilon^{2}\left(\varepsilon^{2}+\left\|\mathbf{w}_{\varepsilon}\right\|_{L^{\infty}(\partial \Omega)}\right),
$$

and hence

$$
\left\|\mathbf{w}_{\varepsilon}\right\|_{L^{\infty}(\partial \Omega)} \leq C \varepsilon^{2}\left(1+\left\|\mathbf{w}_{\varepsilon}\right\|_{L^{\infty}(\partial \Omega)}\right) .
$$

So, if $\varepsilon$ is small enough, then

$$
\left\|\mathbf{w}_{\varepsilon}\right\|_{L^{\infty}(\partial \Omega)} \leq C \varepsilon^{2} .
$$

It then follows from (4.24) that

$$
\mathbf{w}_{\varepsilon}(\mathbf{x})=\frac{\pi \varepsilon^{2}}{E} \frac{\partial}{\partial \nu_{\mathbf{y}}} \mathbf{N}(\mathbf{x}, 0) \frac{\partial \mathbf{u}_{0}}{\partial \nu}(0)+O\left(\varepsilon^{4}\right) .
$$

We obtain the following theorem.

Theorem 4.1. Suppose that $\gamma_{\varepsilon}$ is a linear crack of size $\varepsilon$ and $\mathbf{z}$ is the center of $\gamma_{\varepsilon}$. Then the solution to (2.2) has the following asymptotic expansion:

$$
\left(\mathbf{u}_{\varepsilon}-\mathbf{u}_{0}\right)(\mathbf{x})=\left.\frac{\pi \varepsilon^{2}}{E} \frac{\partial \mathbf{N}}{\partial \nu_{\mathbf{y}}}(\mathbf{x}, \mathbf{y})\right|_{\mathbf{y}=\mathbf{z}} \frac{\partial \mathbf{u}_{0}}{\partial \nu}(\mathbf{z})+O\left(\varepsilon^{4}\right)
$$

uniformly on $\mathbf{x} \in \partial \Omega$. Here $E$ is the Young's modulus.

It is worth emphasizing that in (4.27) the error is $O\left(\varepsilon^{4}\right)$ and the $\varepsilon^{3}$-term vanishes. One can see from the derivation of (4.27) that the $\varepsilon^{3}$-term vanishes because $\gamma_{\varepsilon}$ is a line segment. If it is a curve, then we expect that the $\varepsilon^{3}$-term does not vanish. We also emphasize that (4.27) is a point-wise asymptotic formula, and it can be used to design algorithms to reconstruct cracks from boundary measurements. We can also integrate this formula against the traction $\mathrm{g}$ to obtain the asymptotic formula for the perturbation of the elastic energy as we do in the next section.

Similarly, if we consider the Dirichlet problem

$$
\begin{cases}\nabla \cdot \sigma\left(\mathbf{u}_{\varepsilon}\right)=0 & \text { in } \Omega \backslash \bar{\gamma}_{\varepsilon} \\ \mathbf{u}_{\varepsilon}=\mathbf{f} & \text { on } \partial \Omega \\ \sigma\left(\mathbf{u}_{\varepsilon}\right) \mathbf{e}^{\perp}=0 & \text { on } \gamma_{\varepsilon}\end{cases}
$$

and denote the Green function of Lamé system in $\Omega$ by $\mathbf{G}$, then we get the following asymptotic expansion of its solution $\mathbf{u}_{\varepsilon}$. 
Theorem 4.2. Suppose that $\gamma_{\varepsilon}$ is a linear crack of size $\varepsilon$, located at $\mathbf{z}$. Then the solution to (4.28) has the following asymptotic expansion:

$$
\frac{\partial}{\partial \nu}\left(\mathbf{u}_{\varepsilon}-\mathbf{u}_{0}\right)(\mathbf{x})=\left.\frac{\pi \varepsilon^{2}}{E} \frac{\partial^{2} \mathbf{G}}{\partial \nu_{\mathbf{x}} \partial \nu_{\mathbf{y}}}(\mathbf{x}, \mathbf{y})\right|_{\mathbf{y}=\mathbf{z}} \frac{\partial \mathbf{u}_{0}}{\partial \nu}(\mathbf{z})+O\left(\varepsilon^{4}\right)
$$

uniformly on $\mathbf{x} \in \partial \Omega$.

\section{Topological derivative of the potential energy}

The elastic potential energy functional of the cracked body is given by (2.7), while without the crack the energy functional is given by

$$
J\left[\mathbf{u}_{0}\right]=-\frac{1}{2} \int_{\Omega} \sigma\left(\mathbf{u}_{0}\right): \nabla^{s} \mathbf{u}_{0} .
$$

By the divergence theorem we have

$$
J_{\varepsilon}\left[\mathbf{u}_{\varepsilon}\right]-J\left[\mathbf{u}_{0}\right]=-\frac{1}{2} \int_{\partial \Omega}\left(\mathbf{u}_{\varepsilon}-\mathbf{u}_{0}\right) \cdot \mathbf{g} d \sigma .
$$

Thus we obtain from (4.27)

$$
J_{\varepsilon}\left[\mathbf{u}_{\varepsilon}\right]-J\left[\mathbf{u}_{0}\right]=-\left.\frac{\pi \varepsilon^{2}}{2 E} \frac{\partial \mathbf{u}_{0}}{\partial \nu}(\mathbf{z}) \frac{\partial}{\partial \nu_{\mathbf{y}}} \int_{\partial \Omega} \mathbf{N}(\mathbf{x}, \mathbf{y}) \mathbf{g}(\mathbf{x}) d \sigma(\mathbf{x})\right|_{\mathbf{y}=\mathbf{z}}+O\left(\varepsilon^{4}\right) .
$$

Since

$$
\mathbf{u}_{0}(\mathbf{y})=\int_{\partial \Omega} \mathbf{N}(\mathbf{x}, \mathbf{y}) \mathbf{g}(\mathbf{x}) d \sigma(\mathbf{x}), \quad \mathbf{y} \in \Omega
$$

we have

$$
J_{\varepsilon}\left[\mathbf{u}_{\varepsilon}\right]-J\left[\mathbf{u}_{0}\right]=-\frac{\pi \varepsilon^{2}}{2 E}\left|\frac{\partial \mathbf{u}_{0}}{\partial \nu}(\mathbf{z})\right|^{2}+O\left(\varepsilon^{4}\right) .
$$

We may write (5.3) in terms of the stress intensity factors. The (normalized) stress intensity factors $K_{I}$ and $K_{I I}$ are defined by

$$
K_{I}\left(\mathbf{u}_{0}, \mathbf{e}\right):=\sigma\left(\mathbf{u}_{0}\right) \mathbf{e}^{\perp} \cdot \mathbf{e}^{\perp} \quad \text { and } \quad K_{I I}\left(\mathbf{u}_{0}, \mathbf{e}\right):=\sigma\left(\mathbf{u}_{0}\right) \mathbf{e}^{\perp} \cdot \mathbf{e} .
$$

So, we have

$$
\sigma\left(\mathbf{u}_{0}\right) \mathbf{e}^{\perp}=K_{I} \mathbf{e}^{\perp}+K_{I I} \mathbf{e}
$$

and hence

$$
\left|\frac{\partial \mathbf{u}_{0}}{\partial \nu}(\mathbf{z})\right|^{2}=\left|\sigma\left(\mathbf{u}_{0}\right) \mathbf{e}^{\perp}\right|^{2}=K_{I}^{2}+K_{I I}^{2} .
$$

We obtain the following result.

Theorem 5.1. We have

$$
J_{\varepsilon}\left[\mathbf{u}_{\varepsilon}\right]-J\left[\mathbf{u}_{0}\right]=-\frac{\pi \varepsilon^{2}}{2 E}\left(K_{I}^{2}+K_{I I}^{2}\right)+O\left(\varepsilon^{4}\right)
$$

as $\varepsilon \rightarrow 0$. 
The topological derivative $D_{T} J_{\varepsilon}(\mathbf{z})$ of the potential energy is defined by [23, 24]

$$
D_{T} J_{\varepsilon}(\mathbf{z}):=\lim _{\varepsilon \rightarrow 0}\left(\frac{1}{\rho^{\prime}(\varepsilon)} \frac{d}{d \varepsilon} J_{\varepsilon}\right)
$$

where $\rho(\varepsilon)=\pi \varepsilon^{2}$. So, one can immediately see from (5.7) that

$$
D_{T} J_{\varepsilon}(\mathbf{z})=-\frac{1}{2 E}\left(K_{I}^{2}+K_{I I}^{2}\right) .
$$

This formula is in accordance with the one obtained by Novotny et al in [22] (see also [26]). In fact, in those papers the plane strain and the plain stress problems are considered, and (5.9) is the formula for the latter problem.

\section{Acknowledgement}

Authors would like to thank André Novotny for helpful comments on this paper.

\section{A Derivation of $(\underline{3.3})$}

The Kelvin matrix (2.12) can be rewritten as

$$
\Phi_{i j}(\mathbf{x}-\mathbf{y})=\lambda^{\prime} \delta_{i j} \log |\mathbf{x}-\mathbf{y}|+\mu^{\prime}\left(x_{i}-y_{i}\right) \frac{\partial \log |\mathbf{x}-\mathbf{y}|}{\partial y_{j}}, \quad i, j=1,2,
$$

where

$$
\lambda^{\prime}=\frac{\lambda+3 \mu}{4 \pi \mu(\lambda+2 \mu)}, \quad \mu^{\prime}=\frac{\lambda+\mu}{4 \pi \mu(\lambda+2 \mu)} .
$$

Using the operator $T(\partial)$ defined by (3.2) one can see that

$$
\frac{\partial \Phi}{\partial \nu_{\mathbf{y}}}(\mathbf{x}-\mathbf{y})=\left(T\left(\partial_{\mathbf{y}}\right) \Phi(\mathbf{x}-\mathbf{y})\right)^{T}
$$

or

$$
\left(\frac{\partial \Phi}{\partial \nu_{\mathbf{y}}}(\mathbf{x}-\mathbf{y})\right)_{k j}:=\sum_{l=1}^{2} T_{j l}\left(\partial_{\mathbf{y}}\right) \Phi_{l k}(\mathbf{x}-\mathbf{y}), \quad k, j=1,2 .
$$

We use the formulas

$$
\begin{aligned}
\frac{\partial}{\partial y_{i}} \log |\mathbf{x}-\mathbf{y}| & =-\frac{x_{i}-y_{i}}{|\mathbf{x}-\mathbf{y}|^{2}}, \\
\frac{\partial^{2}}{\partial y_{i}^{2}} \log |\mathbf{x}-\mathbf{y}| & =-2 \frac{\left(x_{i}-y_{i}\right)^{2}}{|\mathbf{x}-\mathbf{y}|^{4}}+\frac{1}{|\mathbf{x}-\mathbf{y}|^{2}}, \\
\frac{\partial^{2}}{\partial y_{i} \partial y_{j}} \log |\mathbf{x}-\mathbf{y}| & =-2 \frac{\left(x_{i}-y_{i}\right)\left(x_{j}-y_{j}\right)}{|\mathbf{x}-\mathbf{y}|^{4}} \quad \text { if } i \neq j .
\end{aligned}
$$


By (A.3), we have

$$
\begin{aligned}
\left(\frac{\partial \Phi}{\partial \nu_{\mathbf{y}}}\right)_{11}= & \left((\lambda+2 \mu) n_{1} \frac{\partial}{\partial y_{1}}+\mu n_{2} \frac{\partial}{\partial y_{2}}\right)\left(\lambda^{\prime} \log |\mathbf{x}-\mathbf{y}|+\mu^{\prime}\left(x_{1}-y_{1}\right) \frac{\partial \log |\mathbf{x}-\mathbf{y}|}{\partial y_{1}}\right) \\
& +\left(\mu n_{2} \frac{\partial}{\partial y_{1}}+\lambda n_{1} \frac{\partial}{\partial y_{2}}\right) \mu^{\prime}\left(x_{1}-y_{1}\right) \frac{\partial \log |\mathbf{x}-\mathbf{y}|}{\partial y_{2}} \\
= & \lambda^{\prime}(\lambda+2 \mu) n_{1} \frac{\partial \log |\mathbf{x}-\mathbf{y}|}{\partial y_{1}}+\lambda^{\prime} \mu n_{2} \frac{\partial \log |\mathbf{x}-\mathbf{y}|}{\partial y_{2}} \\
& +\mu^{\prime}(\lambda+2 \mu) n_{1}\left(-\frac{\partial \log |\mathbf{x}-\mathbf{y}|}{\partial y_{1}}+\left(x_{1}-y_{1}\right) \frac{\partial^{2} \log |\mathbf{x}-\mathbf{y}|}{\partial y_{1}{ }^{2}}\right) \\
& -\mu \mu^{\prime} n_{2} \frac{\partial \log |\mathbf{x}-\mathbf{y}|}{\partial y_{2}}+2 \mu \mu^{\prime} n_{2}\left(x_{1}-y_{1}\right) \frac{\partial^{2} \log |\mathbf{x}-\mathbf{y}|}{\partial y_{1} \partial y_{2}} \\
& +\lambda \mu^{\prime}\left(x_{1}-y_{1}\right) \frac{\partial^{2} \log |\mathbf{x}-\mathbf{y}|}{\partial y_{2}{ }^{2}} .
\end{aligned}
$$

Since $\Delta \log |\mathbf{x}-\mathbf{y}|=0$ for $\mathbf{x} \neq \mathbf{y}$, we have

$$
\begin{aligned}
\left(\frac{\partial \Phi}{\partial \nu_{\mathbf{y}}}\right)_{11}= & (\lambda+2 \mu)\left(\lambda^{\prime}-\mu^{\prime}\right) n_{1} \frac{\partial \log |\mathbf{x}-\mathbf{y}|}{\partial y_{1}}+\mu\left(\lambda^{\prime}-\mu^{\prime}\right) n_{2} \frac{\partial \log |\mathbf{x}-\mathbf{y}|}{\partial y_{2}} \\
& +2 \mu \mu^{\prime}\left(n_{1}\left(x_{1}-y_{1}\right) \frac{\partial^{2} \log |\mathbf{x}-\mathbf{y}|}{\partial y_{1}^{2}}+n_{2}\left(x_{1}-y_{1}\right) \frac{\partial^{2} \log |\mathbf{x}-\mathbf{y}|}{\partial y_{1} \partial y_{2}}\right) .
\end{aligned}
$$

Since

$$
(\lambda+2 \mu)\left(\mu^{\prime}-\lambda^{\prime}\right)+2 \mu \mu^{\prime}=-\frac{\mu}{2 \pi(\lambda+2 \mu)}=\mu\left(\mu^{\prime}-\lambda^{\prime}\right),
$$

we obtain

$$
\left(\frac{\partial \Phi}{\partial \nu_{\mathbf{y}}}\right)_{11}=\left[\mu\left(\mu^{\prime}-\lambda^{\prime}\right)-4 \mu \mu^{\prime} \frac{\left(x_{1}-y_{1}\right)^{2}}{|\mathbf{x}-\mathbf{y}|^{2}}\right] \sum_{l=1}^{2} n_{l} \frac{x_{l}-y_{l}}{|\mathbf{x}-\mathbf{y}|^{2}}
$$

Similarly, we can compute

$$
\begin{aligned}
\left(\frac{\partial \Phi}{\partial \nu_{\mathbf{y}}}\right)_{12}= & \left(\lambda n_{2} \frac{\partial}{\partial y_{1}}+\mu n_{1} \frac{\partial}{\partial y_{2}}\right)\left(\lambda^{\prime} \log |\mathbf{x}-\mathbf{y}|+\mu^{\prime}\left(x_{1}-y_{1}\right) \frac{\partial \log |\mathbf{x}-\mathbf{y}|}{\partial y_{1}}\right) \\
& +\left(\mu n_{1} \frac{\partial}{\partial y_{1}}+(\lambda+2 \mu) n_{2} \frac{\partial}{\partial y_{2}}\right) \mu^{\prime}\left(x_{1}-y_{1}\right) \frac{\partial \log |\mathbf{x}-\mathbf{y}|}{\partial y_{2}} \\
= & \lambda\left(\lambda^{\prime}-\mu^{\prime}\right) n_{2} \frac{\partial \log |\mathbf{x}-\mathbf{y}|}{\partial y_{1}}+\mu\left(\lambda^{\prime}-\mu^{\prime}\right) n_{1} \frac{\partial \log |\mathbf{x}-\mathbf{y}|}{\partial y_{2}} \\
& +2 \mu \mu^{\prime} n_{1}\left(x_{1}-y_{1}\right) \frac{\partial^{2} \log |\mathbf{x}-\mathbf{y}|}{\partial y_{1} \partial y_{2}}+2 \mu \mu^{\prime} n_{2}\left(x_{1}-y_{1}\right) \frac{\partial^{2} \log |\mathbf{x}-\mathbf{y}|}{\partial y_{2}^{2}}
\end{aligned}
$$


Since $\lambda\left(\mu^{\prime}-\lambda^{\prime}\right)+2 \mu \mu^{\prime}=\mu\left(\lambda^{\prime}-\mu^{\prime}\right)$, we have

$$
\begin{aligned}
\left(\frac{\partial \Phi}{\partial \nu_{\mathbf{y}}}\right)_{12}= & {\left[\lambda\left(\mu^{\prime}-\lambda^{\prime}\right)+2 \mu \mu^{\prime}\right] n_{2} \frac{x_{1}-y_{1}}{|\mathbf{x}-\mathbf{y}|^{2}}+\mu\left(\mu^{\prime}-\lambda^{\prime}\right) n_{1} \frac{x_{2}-y_{2}}{|\mathbf{x}-\mathbf{y}|^{2}} } \\
& -4 \mu \mu^{\prime} n_{1} \frac{\left(x_{1}-y_{1}\right)^{2}\left(x_{2}-y_{2}\right)}{|\mathbf{x}-\mathbf{y}|^{4}}-4 \mu \mu^{\prime} n_{2} \frac{\left(x_{1}-y_{1}\right)\left(x_{2}-y_{2}\right)^{2}}{|\mathbf{x}-\mathbf{y}|^{4}} \\
= & -4 \mu \mu^{\prime} \frac{\left(x_{1}-y_{1}\right)\left(x_{2}-y_{2}\right)}{|\mathbf{x}-\mathbf{y}|^{2}} \sum_{l=1}^{2} n_{l} \frac{x_{l}-y_{l}}{|\mathbf{x}-\mathbf{y}|^{2}} \\
& -\mu\left(\mu^{\prime}-\lambda^{\prime}\right)\left[\frac{n_{2}\left(x_{1}-y_{1}\right)-n_{1}\left(x_{2}-y_{2}\right)}{|\mathbf{x}-\mathbf{y}|^{2}}\right] .
\end{aligned}
$$

We also have

$$
\begin{aligned}
\left(\frac{\partial \Phi}{\partial \nu_{\mathbf{y}}}\right)_{22} & =\left(\lambda n_{2} \frac{\partial}{\partial y_{1}}+\mu n_{1} \frac{\partial}{\partial y_{2}}\right) \mu^{\prime}\left(x_{2}-y_{2}\right) \frac{\partial \log |\mathbf{x}-\mathbf{y}|}{\partial y_{1}} \\
& +\left(\mu n_{1} \frac{\partial}{\partial y_{1}}+(\lambda+2 \mu) n_{2} \frac{\partial}{\partial y_{2}}\right)\left(\lambda^{\prime} \log |\mathbf{x}-\mathbf{y}|+\mu^{\prime}\left(x_{2}-y_{2}\right) \frac{\partial \log |\mathbf{x}-\mathbf{y}|}{\partial y_{2}}\right) \\
& =\left[\mu\left(\mu^{\prime}-\lambda^{\prime}\right)-4 \mu \mu^{\prime} \frac{\left(x_{2}-y_{2}\right)^{2}}{|\mathbf{x}-\mathbf{y}|^{2}}\right] \sum_{l=1}^{2} n_{l} \frac{x_{l}-y_{l}}{|\mathbf{x}-\mathbf{y}|^{2}}
\end{aligned}
$$

This proves (3.3).

\section{References}

[1] H. Ammari, P. Calmon, and E. Iakovleva, Direct elastic imaging of a small inclusion, SIAM J. Imaging Sci., 1 (2008), 169-187.

[2] H. Ammari, J. Garnier, V. Jugnon, and H. Kang, Direct reconstruction methods in ultrasound imaging of small anomalies. Lecture Notes in Mathematics, Volume 2035, 31-55, Springer-Verlag, 2011.

[3] H. Ammari, J. Garnier, H. Kang, W.K. Park, and K. Sølna, Imaging schemes for perfectly conducting cracks, SIAM J. Appl. Math., 71 (2011), 68-91.

[4] H. Ammari, J. Garnier, and K. Sølna, A statistical approach to target detection and localization in the presence of noise, Waves in Random and Complex Media, 22 (2012), 40-65.

[5] H. Ammari and H. Kang, Reconstruction of small inhomogeneities from boundary measurements, Lecture Notes in Mathematics 1846, Springer-Verlag (2004).

[6] H. Ammari and H. Kang, Polarization and moment tensors with applications to inverse problems and effective medium theory, Applied Mathematical Sciences, Vol. 162, Springer-Verlag, New York, 2007.

[7] H. Ammari, H. Kang and H. Lee, Layer potential techniques in spectral analysis, Math. Surveys Monogr., Vol. 153, AMS, Providence, RI, 2009. 
[8] H. Ammari, H. Kang, H. Lee and W-K Park, Asymptotic imaging of perfectly conducting cracks, SIAM J. Sci. Comput., 32 (2010), 894-922.

[9] H. Ammari, H. Kang, G. Nakamura and K. Tanuma, Complete asymptotic expansions of solutions of the system of elastostatics in the presence of an inclusion of small diameter and detection of an inclusion, J. Elasticity 67 (2002), 97-129.

[10] E. Beretta and E. Francini, An asymptotic formula for the displacement field in the presence of thin elastic inhomogeneities, SIAM J. Math. Anal., 38 (2006), 1249-1261.

[11] E. Beretta, E. Francini, E. Kim, and J.-Y. Lee, Algorithm for the determination of a linear crack in an elastic body from boundary measurements, Inverse Problems 26 (2010), 085015.

[12] E. Beretta, E. Francini, and S. Vessella, Determination of a linear crack in an elastic body from boundary measurements-Lipschitz stability, SIAM J. Math. Anal., 40 (2008), 984-1002.

[13] B.E. Dahlberg, C.E. Kenig, and G. Verchota, Boundary value problem for the systems of elastostatics in Lipschitz domains, Duke Math. Jour., 57 (1988), 795-818.

[14] N.I. Ioakimidis, Application of finite-part integrals to the singular integral equations of crack problems in plane and three-dimensional elasiticity, Acta mech. 45 (1982), $31-47$.

[15] H. Kang, E. Kim, and J.-Y. Lee, Identification of elastic inclusions and elastic moment tensors by boundary measurements, Inverse Problems 19 (2003), 703-724.

[16] H. Kang, E. Kim, and J.-Y. Lee, Numerical reconstruction of a cluster of small elastic inclusions, Inverse Problems, 23 (2007), 2311-2324.

[17] V.D. Kupradze, Three-dimensional problems of the mathematical theory of elasticity and thermoelasticity, North-Holland, Amsterdam, 1979.

[18] P.A. Martin and F.J. Rizzo, On boundary integral equations for crack problems, Proc. R. Soc. Lond. A, 421 (1989), 341-355.

[19] P.A. Martin, Exact solution of a simple hypersingular integral equation, J. Int. Eqs. Appl., 4 (1992), 197-204.

[20] P.A. Martin and F.J. Rizzo, Hypersingular integrals: how smooth must the density be?, Int. J. Numer. Meth Engng., 39 (1996), 687-704.

[21] N.I. Muskhelishvili, Singular integral equations, Noordhoff, Groningen, 1953.

[22] A.A. Novotny, R.A. Feijóo, E. Taroco and C. Padra, Topological sensitivity analysis, Comput. Methods Appl. Mech. Engrg., 192 (2003), 803-829.

[23] J. Sokolowski and A. Żochowski, On the topological derivative in shape optimization, SIAM J. Control Optim., 37 (1999), 1251-1272. 
[24] J. Sokolowski and A. Żochowski, Topological derivatives of shape functional for elasticity systems, Int. Ser. Num. Math. 139 (2001), 231-244.

[25] F.G. Tricomi, Integral Equations, Interscience, New York, 1957.

[26] N. Van Goethem and A.A. Novotny, Crack nucleation sensitivity analysis, Math. Meth. Appl. Sci. 33 (2010), 1978-1994.

[27] P.P. Zabreyko et al., Integral equations: A reference text, Noordhoff, Leyden, 1975. 